\title{
Some Results Concerning Generalized Positive Boolean Dependencies in Relational Database
}

\author{
Luong Nguyen Hoang Hoa
}

\begin{abstract}
This paper presents some concepts of Generalized Positive Boolean Dependencies (GPBDs) in relational database. Positive formulas means logic formulas formed by variables, constants $0 / 1$ which is true if all variables are true. In fact, it shows that relational database expanded by Generalized Positive Dependencies can process complex database constraints, and traditional dependency is one case of Generalized Positive Dependencies. The first section of this paper is about some basic concepts and the need of Generalized Positive Dependencies in particular, major results involved in this dependency such as equivalence theorem among three types of reasoning for all logical dependency classes: reasoning in the world of all relations, reasoning in the world of 2-tuple relations and reasoning of propositional logic. It also shows that functional dependencies, strong, weak and dual dependencies are ones of cases of Generalized Positive Boolean dependencies. Through equivalence theorem, membership problem can be solved. It defines whether Generalized positive Boole dependency can be derived from set of Generalized positive Boole dependencies or not. The last section presents an algorithm to solve the above problems in general.
\end{abstract}

Index Terms-Positive boolean dependency, generalized positive boolean dependencies; equivalence theorem.

\section{INTRODUCTION}

In 1970, Codd [1] introduced a model of relational database and functional concepts to reflect semantics of data in the real world. One of the major results of the theory of functional dependencies was demonstrated through the equivalence theorem. This theorem affirmed equivalence between reasoning in the world of all relations, reasoning in the world of 2-tuple relations and reasoning of propositional logic.

Applying equivalence theorem helps us solve membership problems, in particular defining whether a constraint can be derived from a set of constraints through changing form of Boolean formulas. In 1980s, the concept of positive Boolean dependencies was expanded from the concept of relational dependencies by separated research groups of Berman, Blok [2] and Sagiv, Delobel et al [3], including constraints described through a positive Boolean formula which are true formulations while all variables are one. In description of functional dependencies and positive Boolean dependencies, a value comparison of attributes was still an equational comparison. For positive Boolean dependencies, the equivalence theorem still conserved its effectiveness. In 1992, Nguyen Xuan Huy and Le Thi Thanh [4] expanded

Manuscript received May, 6, 2011; revised June 31, 2011.

Luong Nguyen Hoang Hoa is with the Department if Information Technology, Posts and Telecommunication Institute of Technology, Vietnam (hoaln79@gmail.com). positive Boolean dependencies into generalized positive Boolean dependencies through replacing equational comparison with binary relation with three properties of reflexitivity, symmetric and partiality. For generalized positive Boole dependencies, the equivalence theorem still remains effective. In 1994, Le Duc Minh, Vu Ngoc Loan and Nguyen Xuan Huy [5] continued to develop generalized positive Boolean dependencies into multi-valued positive Boole dependencies conserving effectiveness of the equivalence theorem.

Class of functional dependencies and almost high dependencies developed after that were all based on equational relation as comparing values of attributes appearing in tuples. In reality, in addition to comparison between equations, there are other forms of comparison, expanding the concept of comparison between these forms that may allow managing data more finely. We consider several examples as follows:

1- As managing printers, choosing size of printed papers depends on the length of lines of data, not on the content of data. Two lines of data which have the same length will be printed on types of paper with the same size. Let us consider two attributes in R relation: D - line of data and S - size of printed paper. Len(d) - length of the number of characters of data line $d$, Len(D) $\rightarrow S$ dependency has wider semantics than $\mathrm{D} \rightarrow \mathrm{S}$ dependency.

\begin{tabular}{ll}
\multicolumn{2}{c}{ TABLE I: R } \\
\hline \hline $\mathrm{D}$ & $\mathrm{S}$ \\
\hline Tran Thanh & $\mathrm{S} 1$ \\
Nguyen Thai Van & $\mathrm{S} 3$ \\
Vu Thanh Thuy & $\mathrm{S} 2$ \\
Le Danh Tuyen & $\mathrm{S} 2$ \\
\hline \hline
\end{tabular}

2. In arithmetic, height of integer $n, H(n)$ is total of that number, for example $\mathrm{H}(2004)=\mathrm{H}(123)=6$.

If we classify numbers based on their height, two different numbers may be in the same class. Thus, $\mathrm{H}(\mathrm{N}) \rightarrow$ CLASS dependency has wider semantics than $\mathrm{N} \rightarrow$ CLASS dependency.

\begin{tabular}{ll}
\multicolumn{2}{c}{ TABLE II: S } \\
\hline \hline $\mathrm{N}$ & CLASS \\
\hline 2004 & 6 \\
123 & 6 \\
816 & 15 \\
\hline \hline
\end{tabular}

3. Russian chemist Mendelev pointed out and classified chemical elements by the number of class of electron as well as the number of electron in their atom structure. Two elements may be different, but if they have the same number of electron classes or/and the same number of free electron, 
they will have some of the same certain properties and are therefore in the same group and so on.

Along with advantages of science and technology, dependencies forms above have been appearing much more in fields of genetics, physics, molecule biology, material technology and so on. Need for a mathematical tool to help describe and reflect those dependency forms in database is urgent and is natural as well.

\section{BASIC CONCEPTS AND RESULTS}

The basic concepts of relational database theory are presented detailed in [6], [7]. In this paper we use the following notation.

Let $U=\left\{x_{1}, \ldots, x_{n}\right\}$ be a finite set of Boole variables, $B$ is a Boolean values, $B=\{0,1\}$. Then the Boolean formulas also known as logic formulas built on the constants $0 / 1$, variables and logical connectives $\vee, \wedge$, and $\rightarrow$ [8], [9].

Let $\mathrm{L}(\mathrm{U})$ be the set of all Boolean formulas built on the set of variables $\mathrm{U}$. Each element vector $0 / 1, v=\left(\mathrm{v}_{1}, \ldots, \mathrm{v}_{\mathrm{n}}\right)$ in space $B^{n}=B \times B \times \ldots \times B$ is called a value assignment. Each Boolean formulas $f \in L(U), f(v)=f\left(v_{l}, \ldots, v_{n}\right)$ is the value of formula $f$ for the assignment of $\mathrm{v}$. We consider to two values assignment: unit assignment $e=(1,1, \ldots, 1)$ and zero assignment $z=(0,0, \ldots, 0)$.

For $F=\left\{f_{1}, f_{2}, \ldots, f_{m}\right\} \subseteq L(U), F$ is considered as a formula $F=f_{1} \wedge f_{2} \wedge \ldots \wedge \mathrm{f}_{m}$. Then each value assignment $v$, the truth value of $F$ will be calculated as $F(v)=f_{1}(v) \wedge f_{2}(v) \wedge \ldots$ $\wedge f_{m}(v)$.

Let $f$ be a formula in $F$. Truth table of $f$, denoted by $T_{f}$,

$T_{f}=\left\{v \in \mathscr{B}^{n} \mid f(v)=1\right\}$. For a finite subset $\mathrm{F}$, we denote $T_{F}=\bigcap_{f \in F} T_{f}$.

Definition 2.1: Let $f$ and $g$ be two Boolean formulas. $f$ implies $g$ (logic inference), written $f=g$ if $T_{f} \subseteq T_{g}$. $f$ and $g$ are equivalent, $f \equiv g$, if $T_{f}=T_{g}$. For $F, G \subseteq L(U)$, $F \vDash G$ if $T_{F} \subseteq T_{G}$, and $F \equiv G$, if $T_{F}=T_{G}$.

A formula $f \in L(U)$ is called positive Boolean formula if $f(e)=1$, where $e=(1, \ldots . ., 1)$ is the valuation that consists of all 1. Let $P(U)$ denote all positive Boolean formulas on $U$. We know that $P(U)$ is equivalent to the set of all formulas that can be built using the connectives $\wedge, \vee, \rightarrow$ and constant 1 [2], [8].

\section{Example 2.1}

1. $A \wedge B,(A \wedge B) \vee(C \wedge B),(A \wedge B) \vee((C \wedge B) \rightarrow B) \vee C \vee 1$ are the positive Boolean formulas.

2. Formula $A \wedge(\neg B)$ is not a positive Boolean formula, because when $\mathrm{A}=\mathrm{B}=1$ we have $1 \wedge 0=0$.

Given attributes of a set $U$. Each value domains $d_{i}$ of $A_{i}$, $1 \leq i \leq n$, contains at least 2 elements. For each value domain $d_{i}$, we consider a mapping $\alpha_{i}: d_{i} \times d_{i} \rightarrow B$. We assume that the mappings $\alpha_{i}$ satisfy the follwing properties:

(i) Reflexivity: $\forall a \in d_{i}: \alpha_{i}(a, a)=1$

(ii) Symmetry: $\forall a, b \in d_{i}: \alpha_{i}(a, b)=\alpha_{i}(b, a)$.

(iii) Partiality: $\exists a, b \in d_{i}: \alpha_{i}(a, b)=0$.

It is easy to see that the equality mappings on $d_{i}, \alpha_{i}(a, b)=$ 1 if $a=b$ and $\alpha_{i}(a, b)=0$ otherwise, $a, b \in d_{i}, 1 \leq i \leq n$

Example 2.2: Let $U=\{A, B, C\}$, where $d_{A}$ is the set of positive integers, $d_{B}$ is the set of real numbers and undetermined value $\#, d_{C}$ is the set of words $w$ on a nonempty alphabet $P$, where the length of $\mathrm{w}$ is not greater than $\mathrm{k}, \mathrm{k} \geq 1$. Then, the following mappings $\alpha_{A}, \alpha_{B}$ and $\alpha_{C}$ satisfy three properties (i)-(iii):

$\alpha_{A}(a, b)=1$ if and only if $a$ and $b$ are simultaneously odd or even numbers. Otherwise $\alpha_{A}(a, b)=0$

$\alpha_{B}(a, b)=1$ if and only if $a$ and $b$ are simultaneously real or two undetermined values \#.

$\alpha_{C}(a, b)=1$ if and only if both $a$ and $b$ have the same length.

Suppose $\alpha_{i}$ mappings are determined on each $d_{i}$ value domain of $A_{i}$ attributes in $U=\left\{A_{1}, A_{2}, \ldots, A_{n}\right\}, 1 \leq i \leq n$.

A relation $R$ over $U$ is a subset of $d_{1} \mathrm{x} \ldots \ldots \mathrm{x} d_{n} \in U$. For $u$, $v \in R$, we denote by $\alpha(\mathrm{u}, \mathrm{v})$ the valuation:

$\alpha(u, v)=\alpha_{1}\left(u \cdot A_{1}, v \cdot A_{1}\right), \alpha_{2}\left(u \cdot A_{2}, v \cdot A_{2}\right), \ldots, \alpha_{n}\left(u \cdot A_{n}, v \cdot A_{n}\right)$

The class of all relations over $U$ is denoted by REL(U).

For $R \in \operatorname{REL}(\mathrm{U})$, we denote $T_{R}=\{\alpha(u, v) / u, v \in R\}$.

\section{Example 2.3}

With $A, B$ and $C$ attributes and $\alpha_{A}, \alpha_{B}$ and $\alpha_{C}$ mappings respectively as described in the previous example, consider the following relations $\mathrm{R}$. We have the following truth table $T_{R}$

\begin{tabular}{cccl}
\multicolumn{4}{c}{ TABLE III: R } \\
\hline \hline Bộ & A & B & C \\
\hline t1 & 14 & 7.5 & Vy \\
t2 & 6 & $\#$ & Manh \\
t3 & 1 & 2.0 & Xuan \\
\hline \hline
\end{tabular}

\begin{tabular}{cccc}
\multicolumn{4}{c}{ TABLE IV: $\mathrm{T}_{\mathrm{R}}$} \\
\hline \hline & $\mathrm{A}$ & $\mathrm{B}$ & $\mathrm{C}$ \\
\hline$\alpha(\mathrm{t} 1, \mathrm{t} 1)$ & 1 & 1 & 1 \\
$\alpha(\mathrm{t} 1, \mathrm{t} 2)$ & 1 & 0 & 0 \\
$\alpha(\mathrm{t} 1, \mathrm{t} 3)$ & 0 & 1 & 0 \\
$\alpha(\mathrm{t} 2, \mathrm{t} 3)$ & 0 & 0 & 1 \\
\hline \hline
\end{tabular}

Definition 2.2: Elements of $P(U)$ are called generalized positive Boolean dependency (GPBD) [4].

Definition 2.3: For $R \in \mathrm{REL}(\mathrm{U})$ and $f \in P(U)$, we say $R$ satisfies the GPBD, written $R(f)$, if $T_{R} \subseteq T_{f}$.

Definition 2.4: Let $R \in \mathrm{REL}(\mathrm{U})$ and $F \in P(U)$, we say that $R$ satisfies the set of GPBDs $F$, written $R(F)$, if $R(F) \Leftrightarrow \forall f \in$ $F: R(f) \Leftrightarrow T_{R} \subseteq T_{F}$.

Definition 2.5: For $F \subseteq P(U)$ and $f \in P(U), F \vdash f$ means that $\forall R \in R E L(U): R(F) \Rightarrow R(f) . F \quad F_{2} f$ means that $\forall R$ $\in R E L_{2}(U): R(F) \Rightarrow R(f)$. In other word, $F \vdash f$ if and only if for all $R \in R E L(U), T_{R} \subseteq T_{F}$ implies $T_{R} \subseteq T_{\text {f. }}$

\section{Theorem 2.1: (Equivalence Theorem)}

[3] Let $F \subseteq P(U)$ and $f \in P(U)$. The following are equivalent:
(i) $F \neq f$
(ii) $F \vdash f$
(iii) $F F_{2} f$

\section{Proof:}

(i) $\Rightarrow$ (ii) If $F \vDash f$ then $T_{F} \subseteq T_{f}$.

Suppose $R \in R E L(R)$ and $R(F)$. Then $T_{R} \subseteq T_{F}$, so by the transitivity, $T_{R} \subseteq T_{f}$. It follows that $R(f)$ and hence 
$F \vDash f$.

(ii) $\Rightarrow$ (iii) is obvious, because $R E L_{-} 2(U) \subseteq R E L(U)$.

(iii) $\Rightarrow$ (i). Assume (iii) and $t=\left(t_{1}, t_{2}, \ldots, t_{n}\right) \in T_{F}$. We have to show $t \in T_{f}$. For this if $t \neq e$, let us construct a relation $R$ with two tuples $u=\left(u_{1}, u_{2}, \ldots, u_{n}\right)$ and $v=\left(v_{1}, v_{2}, \ldots, v_{n}\right)$ such that $\alpha_{i}\left(u_{i}, v_{i}\right)=t_{i}, 1 \leq i \leq n$. The existance of $u_{i}$ and $v_{i}$ is guaranted by properties (i) - (iii). Thus we have $R \in R E L_{-} 2(U)$ and $T_{R}=\{e, t\}$ where e is defined as $(1, \ldots 1)$. Hence $R(F)$. By (iii), $R(f)$. Therefore $T_{R} \subseteq T_{f}$, so as $t \in T_{f}$ desired.

The following dependencies belong to the family of Positive Boolean Dependencies [6], [10]:

TABLE V: Class of Positive BoOLEAN DEPENDECIES

\begin{tabular}{lcc}
\hline \hline Dependency & Symbol & Positive Boole Formula \\
\hline Functional dependency & $X \rightarrow Y$ & $\wedge X \rightarrow \wedge Y$ \\
Strong dependency & $X(s) \rightarrow Y$ & $\vee X \rightarrow \wedge Y$ \\
Weak dependency & $X(w) \rightarrow Y$ & $\wedge X \rightarrow \vee Y$ \\
$\begin{array}{l}\text { Duality dependency } \\
\begin{array}{l}\text { Multi-valued } \\
\text { dependencies }\end{array}\end{array}$ & $X(d) \rightarrow Y$ & $\vee X \rightarrow \vee Y$ \\
\hline \hline
\end{tabular}

\section{MEMBERSHIP PROBLEM AND UNIFICATION ALGORITHM}

Membership problem can be expressed as follows: Given a relation scheme $\mathrm{a}=(\mathrm{U}, \mathrm{F}), \mathrm{U}$ is a set of attributes and $\mathrm{F}$ is set of GPBDs, $F \subseteq P(U)$. $f$ is a single GPBD, $f \in P(U)$. Decide whether $F$ can imply $f$ or not, written by $F \vDash f[3],[4]$.

The following results give some necessary and sufficient conditions for the dependencies $\wedge \mathrm{X} \rightarrow \wedge \mathrm{Y}, \wedge \mathrm{X} \rightarrow \vee \mathrm{Y}$, $\vee \mathrm{X} \rightarrow \wedge \mathrm{Y}, \vee \mathrm{X} \rightarrow \vee \mathrm{Y}, \wedge \mathrm{X} \rightarrow(\wedge \mathrm{X} \vee \wedge \mathrm{Y})$ to be implied from a set of GPBDs $F$ [8], [9].

From propositional logic we know that $F \vDash g \rightarrow h$ if and only if for all $t \in T_{F}$, either $g(t)=0$ or $h(t)=1$.

$$
F \vDash g \rightarrow h \Leftrightarrow \forall t \in T_{F}: g(t)=0 \vee h(t)=1
$$

By an application of this claim, we have:

\section{Theorem 3.1:}

Let $F$ be a set of GPBDs on $U$, and $X, Y, Z \subseteq U$. Then

1. $F \vDash \wedge X \rightarrow \wedge Y \Leftrightarrow$ $\Leftrightarrow \forall v \in T_{F}:((\exists A \in X: v \cdot A=0) \vee(\forall B \in Y: v \cdot B=1))$

2. $F \vDash \wedge X \rightarrow \vee Y \Leftrightarrow$ $\Leftrightarrow \forall v \in T_{F}:((\exists A \in X: v \cdot A=0) \vee(\exists B \in Y: v \cdot B=1))$

3. $F \vDash \vee X \rightarrow \wedge Y \Leftrightarrow$ $\Leftrightarrow \forall v \in T_{F}:((\forall A \in X: v \cdot A=0) \vee(\forall B \in Y: v \cdot B=1))$

4. $F \vDash \vee X \rightarrow \vee Y \Leftrightarrow$

$$
\Leftrightarrow \forall v \in T_{F}:((\forall A \in X: v \cdot A=0) \vee(\exists B \in Y: v \cdot B=1))
$$

5. $F \vDash \wedge X \rightarrow(\wedge Y) \vee(\wedge Z) \Leftrightarrow$ $\Leftrightarrow \forall v \in T_{F}:((\exists A \in X: v \cdot A=0) \vee(\forall B \in Y: v \cdot B=1) \vee(\forall C \in Z: v \cdot C=1))$

For $U=\left(A_{1}, A_{2}, \ldots, A_{n}\right) \in B^{n} \quad, \quad$ we define $\operatorname{Set}(v)=\{A \in U \mid v \cdot A=1\}$ and for $T \in B^{n}$ we define $\operatorname{Set}(T)=\{\operatorname{Set}(v) \mid v \in T\}$. We get the following result:

\section{Theorem 3.2:}

Let $F$ be a set of GPBDs on $U$, and $X, Y, Z \subseteq U$. Then

1. $F \vDash \wedge X \rightarrow \wedge Y \Leftrightarrow \forall V \in \operatorname{Set}\left(T_{F}\right):(X \subseteq V \Rightarrow Y \subseteq V)$

2. $F=\wedge X \rightarrow \vee Y \Leftrightarrow \forall V \in \operatorname{Set}\left(T_{F}\right):(X \subseteq V \Rightarrow Y \cap V \neq \varnothing)$

3. $F=\vee X \rightarrow \wedge Y \Leftrightarrow \forall V \in \operatorname{Set}\left(T_{F}\right):(X \cap V \neq \varnothing \Rightarrow Y \subseteq V)$

4. $F=\vee X \rightarrow \vee Y \Leftrightarrow \forall V \in \operatorname{Set}\left(T_{F}\right):(X \cap V \neq \varnothing \Rightarrow Y \cap V \neq$ Ø)

$$
\begin{gathered}
\text { 5. } F \vDash \wedge X \rightarrow(\wedge Y) \vee(\wedge Z) \Leftrightarrow \forall V \in \operatorname{Set}\left(T_{F}\right): \\
(X \subseteq V \Rightarrow(Y \subseteq V) \vee(Z \subseteq V))
\end{gathered}
$$

Let $f$ be a GPBD and $F$ be a set of GPBDs on $U$. For $X \subseteq U$, denote by $f \backslash X$ the formula constructed from $f$ by replacing all the occurences of symbols in $X$ by 1 . For $F$ we denote $F \backslash X=\{f \backslash X \mid f \in F\}$.

Note that $F \vDash \wedge X \rightarrow \wedge Y$ if and only if $F \vDash \wedge X \rightarrow A$ for every $A \in Y-X$. Thus $f \backslash X$ and $F \backslash X$ can be considered as formulas on $U-X$.

\section{Theorem 3.3:}

Let $F$ be a GPBD set of the form $\wedge X \rightarrow \wedge Z, Y, Z \subseteq U$, and let $X \subseteq U, A \in U \backslash X$. Then $F \vDash \wedge X \rightarrow A$ on $U$ if and only if $F \backslash X \vDash 1 \rightarrow A$ on $U \backslash X$.

\section{Proof:}

Let $V=U-X$ and $G=F \backslash X$

If: Assume $G \vDash 1 \rightarrow A$ on $V$ and suppose that $x$ is a truth assignment for $F$ on $U$ such that $F(x)=1$ and $\wedge X(x)=1$. From $y$ from $x$ by removing the values $x(B)$ for all $B \in X$. Clearly, $y$ is an assignment on $V$ and $G(y)=1$. Therefore $y(A)=x(A)=1$, since $G \vDash 1-A$. It follows that $F=$ $\wedge X \rightarrow A$.

Only if: Assume $F \vDash \wedge X A$ and suppose that $y$ is atruth assignment for $G$ on $V$ such that $G(y)=1$. Form $y$ from $x$ by adding the values $x(B)=1$ for all $B \in X$. Clearly, $x$ is the assignment on $U$. Moreover, $F(x)=1$ and $\wedge X(x)=1$. Since $F \vDash \wedge X \rightarrow A$, it follows that $y(A)=x(A)=1$. Therefore $G \vDash 1 \rightarrow A$.

Theorem 3.3 gives a basis for the concept of translations of relation shemes. The main purpose of this concept is to transform a given set functional dependencies by removing some attributes that seem to be unimportant for computing several objects in the relational model of databases.

The similar results for differential dependencies were presented in [11].

In the last of this section, we also give an algorithm to 
solve the above problem in general based on some results of algorithm Unification.

From equivalence theorem, the above problem is solved by the following proposition:

$F=f$ if and only if $F \rightarrow f$

Algorithm Unification was introduced first time in [9], after that it was developed and set up in Prolog environment [12].

To prove $E$ positive formula is true, the following steps is performed:

1. Present $\neg E$ in the following form:

$C=C_{1} \wedge C_{2} \wedge \ldots \wedge C_{k}$ (conjunctive normal form), in which $C_{i}$ is sum of all variables and constants $0 / 1$. Repeat unification steps until $C$ cannot be changed: Find $C_{i}$ and $C_{j}$ in form $C_{i}=(p \vee x)$ and $C_{j}=(q \vee \neg x)$

- If finding results is ok then replace $C_{i}$ and

$$
C j \text { in } C \text { by }(p \vee q) \text {. }
$$

2. Conclusion: If $C=\varnothing$ then $E$ formula is proved; else $E$ is not tautology.

\author{
Algorithm Proof \\ Function: \\ Proof $E$ formula \\ Input: \\ E Positive Formula \\ Output: \\ True if $E$ is tautology; vice versa: False \\ Method \\ 1. Take $\neg E$ to conjunctive normal form: \\ $C:=\operatorname{cnf}(\neg E)$; \\ 2. Unification: return(Unification $(C)=\varnothing)$; \\ EndResolution.
}

\section{Algorithm Unification}

\section{Function:}

Unify all elements in positive formula in conjunctive normal form $C$ to maximum

Input:

$$
C=C_{1} \wedge C_{2} \wedge \ldots \wedge C_{k}
$$

\section{Output:}

Formula after unification

Method

while (to be performed) do

Find 2 elements in $C$ in forms

$$
C_{i}=(p \vee x) \text { and } C_{j}=(q \vee \neg x)
$$

if (finding results is ok)

replace $C_{i}$ and $C j$ in $C$ by $(p \vee q)$

else break;

endif;

endwhile;

return $C$;

EndUnification.

To solve this problem, we have some comments :

$$
F \mid f \equiv F \rightarrow f \equiv \neg F \vee f
$$

Let $E \equiv \neg F \vee f, \neg E \equiv \neg(\neg F \vee f) \equiv F \wedge \neg f$

We also know $F$ is set of GBPDs so $F$ is needed to take unification form and perform maximum unification steps. The following Algorithm Reduction performs the above task.

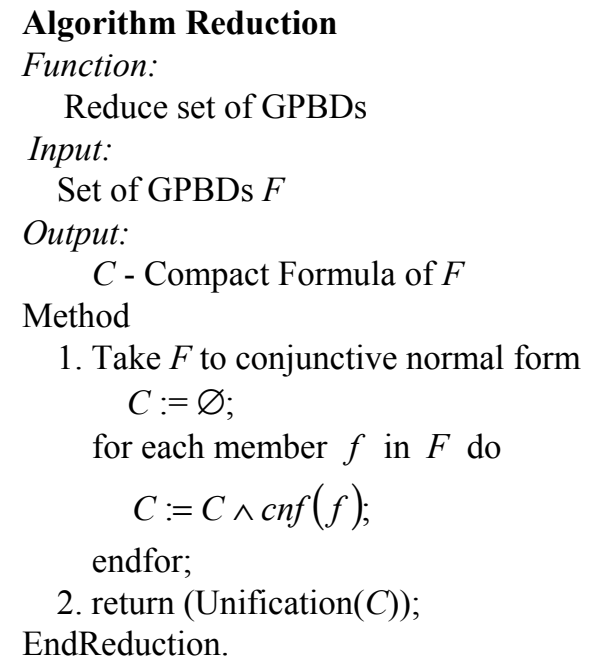

Finally, to solve membership problem $F \vDash f$ we call the following Member algorithm:

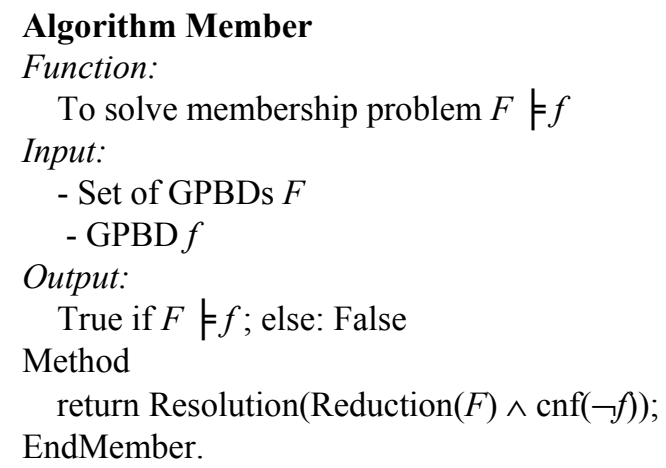

\section{CONCLUSION}

In this paper, we introduce some results concerning Generalized Positive Boolean Dependencies in logical dependency classes. We have proposed algorithm to solve membership problem with Generalized positive Boolean dependency in general. These results can be used to design and manage complex and large-scale databases.

\section{REFERRENCES}

[1] E. F. Codd, "A Relational Model of Data for Large Shared Data Banks," CACM, vol. 13, pp. 377-387, June 1970.

[2] J. Berman and W. J. Blok, "Positive Boolean dependencies," Inf. Processing Letters, vol. 27, pp. 147-150, March 1988

[3] Y. Sagiv, C. Delobel, D. S. Parker, and R. Fagin, "An equivalence between Relational Database Dependencies and a Fragment of Propositional Logic," J. ACM, vol. 28, pp. 435-453, 1981

[4] N. X. Huy and L T. Thanh, "Generalized Positive Boolean Dependencies," J. Inf. Process. Cybern. EIK, vol. 28, pp. 363-370, 1992.

[5] L. D. Minh, V. N. L, and N. X. Huy, "Some Results Concerning Covers in the Class of Multivalued Positive Boolean Dependencies," in Proc.2005 World Scientific Conf, 2005, pp. 119-130.

[6] N. X. Huy, Logic dependencies in database, Institute of Information Technology, Statistical Publishing House, 2006, ch.5.

[7] R. Elmasri and S. B. Navathe, Fundamentals of Database Systems $\left(6^{\text {th }}\right.$ Edition), Addison Wesley, April 2010, ch.3. 
[8] F. Baader and W. Snyder, Handbook of Automated Reasoning, Elsevier Science Publishers, 2001, ch.8.

[9] R. A. owalski, Logic for Problem Solving, North Holland, 1979, ch. 2.

[10] N. X. Huy, Đ. V. Ban, Đ. G. Manh, and N. T. Dung, "The relationship between funtional dependency inference and logical inference," Journal of Computer Science and Cybernetics, vol. 17, no. 4, pp. 11-16, 2001.

[11] S. Song and L. Chen, "Differential Dependencies: Reasoning and Discovery," ACM Trans. Datab. Syst, vol.9, 4, Article 39, March 2011

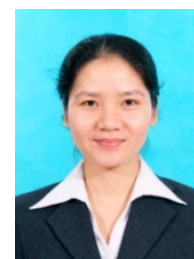

Luong Nguyen Hoang Hoa was born in HaNoi, Vietnam, 1979.

Brief Biographical History:

- 2001, 2005: Bachelor, Master in Information Tchnology of HaNoi National University, Vietnam.

- 2008: Ph.D Student in Posts \& Telecommunications Institute of Technology, Vietnam.

[12] I. Bratko, Prolog Programming for Artificial Intelligence, Addison-Wesley, 3rd Edition, ISBN-10: 0201403757, 2000. 